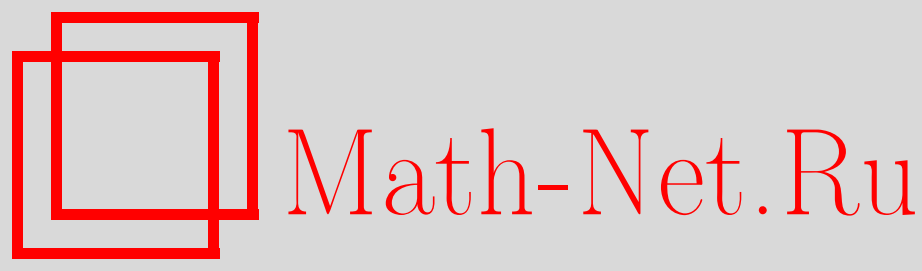

М. В. Комарова, И. С. Кремнев, М. Ю. Налимов, Семейство инстантонов модели Крейчнана с замороженным полем скорости, ТМФ, 2009, том 158, номер 2, 200-213

DOI: https://doi.org/10.4213/tmf6309

Использование Общероссийского математического портала Math-Net.Ru подразумевает, что вы прочитали и согласны с пользовательским соглашением http://www . mathnet.ru/rus/agreement

Параметры загрузки:

IP: 54.198 .187 .58

26 апреля 2023 г., 09:06:16

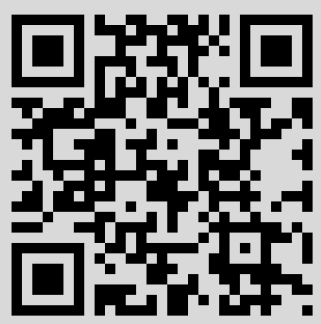




\title{
СЕМЕЙСТВО ИНСТАНТОНОВ МОДЕЛИ КРЕЙЧНАНА С ЗАМОРОЖЕННЫМ ПОЛЕМ СКОРОСТИ
}

\begin{abstract}
Найдено семейство инстантонов для модели Крейчнана с замороженным полем скорости. С их помощью исследована асимптотика высоких порядков рядов теории возмущений, построенных для функции отклика. Показано, что, хотя число диаграмм с ростом порядка теории возмущений растет факториально, ряды теории возмущений имеют конечный, а иногда и бесконечный радиус сходимости. Тем самым опровергнуто распространенное утверждение о том, что тип сходимости рядов можно определять, оценивая число диаграмм в высоких порядках теории возмущений.
\end{abstract}

Ключевые слова: теория возмущений, асимптотика высоких порядков, инстантон, модель Крейчнана, замороженное поле скорости.

\section{1. ВВЕДЕНИЕ}

Применение квантово-полевой теории возмущений приводит, как правило, к медленно сходящимся или даже асимптотическим рядам. В то же время вычисление каждого последующего порядка теории возмущений требует более сложных вычислений. Поэтому для получения численных значений широко используются разнообразные схемы пересуммирования, при выборе которых опираются на информацию о типе сходимости рядов. Эти схемы применяют к очень небольшому отрезку ряда, содержащему два-три, в лучшем случае пять-шесть членов. Так, например, для факториально растущих рядов используют пересуммирование на основе метода Бореля-Лероя [1].

$\mathrm{K}$ сожалению, чаще всего при пересуммировании не используются явно параметры асимптотики высоких порядков разложений. Вместо этого вводятся разнообразные подгоночные параметры и погрешность таких схем всегда остается неизвестной. Конечно, в рамках такого подхода нельзя выяснить, насколько хорошо вычисленные точно порядки теории возмущений соответствуют полученной асимптотике.

\footnotetext{
${ }^{*}$ Санкт-Петербургский государственный университет, Санкт-Петербург, Россия.
} E-mail: komarova1@paloma.spbu.ru, Mikhail.Nalimov@pobox.spbu.ru

${ }^{\dagger}$ Институт аналитического приборостроения РАН, Санкт-Петербург, Россия. E-mail: ilya.kremnev@gmail.com 
Вычисление асимптотики высоких порядков квантово-полевых рядов было впервые предложено в работе [2] и основывается на методе стационарной фазы в функциональном интеграле. При этом должен быть найден инстантон - решение уравнений стационарности. Однако в настоящее время широко распространено утверждение, что для грубой оценки асимптотики высоких порядков достаточно знать лишь количество диаграмм в данном порядке теории возмущений (см., например, [3]).

В динамических полевых моделях, описывающих турбулентные явления, выяснение типа сходимости ряда связано со значительными сложностями. Эти задачи не имеют статического предела, для которого проблема была решена в работах [4], [5]. Как показано в работах [6], [7], если удается ввести переменные Лагранжа, ситуация несколько упрощается. $\mathrm{K}$ таким случаям относится модель турбулентной диффузии Крейчнана. Асимптотика высоких порядков разложений этой модели была вычислена в работе [7]. Оказалось, что ряды имеют конечный радиус сходимости. Спецификой теории возмущений этой модели является то, что из-за дельтаобразного по времени коррелятора скорости многие диаграммы теории возмущений равны нулю, так что количество диаграмм растет степенным образом и действительно определяет в каком-то смысле скорость сходимости ряда.

В настоящей работе мы исследуем асимптотику высоких порядков для модели Крейчнана с замороженным полем скорости, описывающей диффузию жидкости в стационарном случайном поле [3], [8], [9]. Коррелятор поля скорости в этом случае, в отличие от стандартной модели Крейчнана, не зависит от времени, а по координате ведет себя степенным образом, так что число диаграмм теории возмущений растет факториально.

Условием стационарности в данной модели является система нелинейных интегродифференциальных уравнений. Мы покажем, как строить семейство ее решений семейство инстантонов, и получим в аналитическом виде один из них. Проведенный с помощью полученного решения анализ показывает, что тип сходимости рядов теории возмущений определяется разнообразными факторами: исследуемым объектом, выбранным представлением (координатным или импульсным), видом коррелятора скорости, а не только одним числом диаграмм. Мы обнаружили случаи, когда исследуемые ряды имеют конечный и даже бесконечный радиус сходимости.

Вообще говоря, для исследуемых уравнений стационарности априори неизвестной является не только единственность, но и сам факт существования решения. Нам удалось найти семейство решений данных уравнений с наиболее естественной для данной задачи симметрией, причем каждому представителю семейства решений соответствуют свои граничные условия. На самом деле мы не можем ничего утверждать о возможности существования инстантонов с другой симметрией и об их вкладах в асимптотики высоких порядков. К сожалению, это замечание относится и к инстантонному анализу асимптотик высоких порядков разложений и для всех других моделей, включая “классическую” модель $\phi^{4}$.

Однако случай, когда коррелятор поля скорости не зависит не только от времени, но и от координаты, оказывается частным точно решаемым случаем рассматриваемой задачи. Мы будем использовать его, чтобы проверить правильность расчетов.

Работа построена следующим образом. В разделе 2 дано краткое описание модели Крейчнана с замороженным полем скорости, а также приведены корреляторы случайной скорости, которые будут исследоваться далее. В разделе 3 приведено 
точное решение для частного случая рассматриваемой модели. В случае, когда коррелятор поля скорости не зависит от координаты, вычислены точно $N$-й порядок теории возмущений для функции отклика и показано, что соответствующий ряд имеет конечный радиус сходимости. В разделе 4 вводятся переменные Лагранжа и строится действие, для которого в разделе 5 рассматривается метод стационарной фазы. В разделе 6 доказано, что полученная в разделе 5 система уравнений имеет семейство решений - инстантонов. Удается найти первый интеграл движения и с его помощью получить явное решение, позволяющее вычислить тип сходимости рядов упомянутой модели. Выводы приведены в разделе 7. В приложении А показано как наш метод работает в точно решаемом случае. Некоторые справочные формулы приведены в приложении Б.

\section{2. МОДЕЛЬ КРЕЙЧНАНА С ЗАМОРОЖЕННЫМ ПОЛЕМ СКОРОСТИ}

Распространение пассивной скалярной примеси в $d$-мерной вязкой сжимаемой жидкости происходит посредством диффузии и турбулентного перемешивания случайным полем скорости. Описание задачи в рамках модели Крейчнана основывается на стохастическом уравнении

$$
\left(\partial_{t}+g \nabla_{i} V_{i}-\nu \Delta\right) \varphi(x)=\xi(x)
$$

где, как и в работе [10], введена единая пространственно-временная переменная $x \equiv\left\{t, \mathbf{x} \in \mathbb{R}^{d}\right\}, \varphi(x)$ - скалярное поле, описывающее примесь, $\mathbf{V}(\mathbf{x})$ - случайное векторное поле скорости, $\xi(x)$ - случайная сила, $\nu$ - вязкость. Также для компактности введены следующие обозначения:

$$
\partial_{t} \equiv \frac{\partial}{\partial t}, \quad \Delta \equiv \sum_{i=1}^{d} \frac{\partial^{2}}{\partial \mathbf{x}_{i}^{2}}, \quad \nabla_{i} \equiv \frac{\partial}{\partial \mathbf{x}_{i}}
$$

В уравнении (1) и далее подразумевается свертка по повторяющимся векторным индексам. Стандартная теория возмущений приводит к рядам по константе взаимодействия $g$, свойствами которых мы и интересуемся. Для случайных величин $\xi$ и $\mathbf{V}$ предполагаются гауссовы распределения, коррелятор случайной силы $D_{\xi}$ произвольный, коррелятор поля скорости, в отличие от стандартной модели Крейчнана, рассматривается не зависящим от времени $\left\langle\mathbf{V}_{i}(x) \mathbf{V}_{j}\left(x^{\prime}\right)\right\rangle=D_{i j}\left(\mathbf{x}-\mathbf{x}^{\prime}\right)^{1)}$. В импульсном представлении коррелятор имеет степенной вид (см. [8], [9])

$$
D_{i j}^{\mathrm{F}}(\mathbf{q}) \equiv \int d^{d} \mathbf{z} D_{i j}(\mathbf{z}) e^{i \mathbf{q z}}=\lambda_{\mathrm{T}}\left(\delta_{i j}-\frac{q_{i} q_{j}}{q^{2}}\right) \frac{1}{q^{2 \alpha}}+\lambda_{\mathrm{L}} \frac{q_{i} q_{j}}{q^{2}} \frac{1}{q^{2 \alpha}},
$$

а в координатном

$$
D_{i j}(\mathbf{z})=a_{1} \frac{\delta_{i j}}{\mathbf{z}^{2 \beta}}+a_{2} \frac{\mathbf{z}_{i} \mathbf{z}_{j}}{\mathbf{z}^{2 \beta+2}}, \quad \beta=\frac{d}{2}-\alpha,
$$

параметры $a_{1}, a_{2}$ определены в приложении Б.

\footnotetext{
1) Данная модель описывает диффузию в случайных средах [8], [9] и имеет непосредственное отношение к проблеме развитой турбулентности (см., например, [11]).
} 
Стохастическое уравнение (1) с помощью формализма Мартина-Сиггиа-Розе (MSR-формализм) [12] стандартным образом преобразуется в квантово-полевую модель с действием вида

$$
\begin{aligned}
S^{\mathrm{MSR}}= & \int d x d x^{\prime}\left(\frac{\varphi^{\prime}(x) D_{\xi} \varphi^{\prime}\left(x^{\prime}\right)}{2}+\frac{\mathbf{V}_{i}(\mathbf{x}) D_{i j}^{-1}\left(\mathbf{x}-\mathbf{x}^{\prime}\right) \mathbf{V}_{j}\left(\mathbf{x}^{\prime}\right)}{2}\right)+ \\
& +\int d x\left(\varphi^{\prime}(x)\left[\partial_{t}+g \mathbf{V}_{i}(\mathbf{x}) \nabla_{i}-\nu \Delta\right] \varphi(x)\right) .
\end{aligned}
$$

Объектом исследования теперь становятся корреляционные функции. В частности, после стандартных преобразований (см. [10]) функция отклика принимает вид

$$
G\left(x_{2}-x_{1}\right)=\frac{\int \mathcal{D} \mathbf{V} \mathcal{D} \varphi \mathcal{D} \varphi^{\prime} \varphi\left(x_{1}\right) \varphi^{\prime}\left(x_{2}\right) e^{-S^{\mathrm{MSR}}}}{\int \mathcal{D} \mathbf{V} \mathcal{D} \varphi \mathcal{D} \varphi^{\prime} \exp \left(-\left.S\right|_{g=0} ^{\mathrm{MSR}}\right)} .
$$

Знаменатель этого выражения представляет собой нормировочный множитель, соответствующий свободной $(g=0)$ модели.

Для анализа инфракрасного поведения этой модели используется метод ренормализационной группы и регулярные разложения по $\epsilon=2+2 \alpha-d$ [8], [9]. Это приводит к необходимости ренормировки модели при $\epsilon \rightarrow 0$. В настоящей работе мы не рассматриваем инфракрасное поведение модели и механизм ренормировки в высоких порядках теории возмущений (т.е. свойства рядов по параметру $\epsilon$ ). Мы ограничились изучением исходных рядов по константе связи $g$, не преобразованных методом ренормгруппы. Это позволяет нам считать параметр регуляризации $\epsilon$ отличным от нуля и избежать тем самым взаимного влияния больших параметров $N$ и $1 / \epsilon$.

\section{3. ТОЧНО РЕШАЕМЫЙ ЧАСТНЫЙ СЛУЧАЙ: ПОЛЕ V(x) ПОСТОЯННО}

Пространство функций, по которому проводится функциональное интегрирование, определяется типом решаемой задачи. Подробно вопрос о правильном выборе пространства функционального интегрирования для построения теории возмущений рассмотрен в монографии [10]. Обычно в статических задачах интегрирование проводится в пространстве достаточно быстро (экспоненциально) убывающих функций, однако в динамике существуют задачи с другими функциональными пространствами [4].

В частном случае, когда поле скорости рассматриваемой модели принадлежит классу постоянных функций, т.е. не зависит от $t$ и $\mathbf{x}$, задача оказывается точно решаемой. Действительно, функция Грина линейного уравнения диффузии (1) при $g=0$ хорошо известна,

$$
\frac{\Theta\left(t-t^{\prime}\right)}{\left(4 \pi \nu\left(t-t^{\prime}\right)\right)^{d / 2}} \exp \left(-\frac{\left(\mathbf{x}-\mathbf{x}^{\prime}\right)^{2}}{4 \nu\left(t-t^{\prime}\right)}\right)
$$

где $\Theta$ - функция Хевисайда. Функция Грина для произвольных $g$ получается заменой $\mathbf{x} \rightarrow \mathbf{x}+g \mathbf{V} t$. Поэтому уравнение (1) имеет вид

$$
\varphi(x)=\int_{-\infty}^{\infty} d x^{\prime} \frac{\Theta\left(t-t^{\prime}\right)}{\left(4 \pi \nu\left(t-t^{\prime}\right)\right)^{d / 2}} \exp \left(-\frac{\left(\mathbf{x}-\mathbf{x}^{\prime}+g \mathbf{V}\left(t-t^{\prime}\right)\right)^{2}}{4 \nu\left(t-t^{\prime}\right)}\right) \xi\left(x^{\prime}\right) .
$$


Отсюда, после усреднения по всем возможным скоростям с учетом нормировки для функции отклика получаем

$$
G(T, \mathbf{x})=\left\langle\frac{\delta \varphi\left(\xi\left(x_{2}\right)\right)}{\delta \xi\left(x_{1}\right)}\right\rangle=\frac{\Theta(T)(2 \nu T)^{d / 2}}{\left(2 \nu T+D g^{2} T^{2}\right)^{d / 2}} \exp \left(-\frac{\mathbf{x}^{2}}{4 \nu T+2 D g^{2} T^{2}}\right),
$$

где мы ввели трансляционно-инвариантные переменные $\mathbf{x} \equiv \mathbf{x}_{2}-\mathbf{x}_{1}$ и $T \equiv t_{2}-t_{1}$.

$N$-й порядок разложения этой функции по $u \equiv g^{2}$ выражается через гипергеометрическую функцию ${ }_{1} F_{1}\left(N+d / 2, d / 2,-\mathbf{x}^{2} /(4 \nu T)\right)$ (см. приложение Б). Последующее вычисление асимптотического предела при $N \rightarrow \infty$ основано на связи ${ }_{1} F_{1}$ с функцией Уиттекера, для которой соответствующая асимптотика хорошо известна [13]. Таким образом, точное решение имеет вид

$$
\begin{aligned}
G^{[N]}(T, \mathbf{x})= & \frac{\Theta\left(t-t^{\prime}\right)}{\sqrt{\pi}} e^{1-d / 2} e^{-\mathbf{x}^{2} /(8 \nu T)}\left(\frac{\mathbf{x}^{2}}{4 \nu T}\right)^{(1-d) / 4} \times \\
& \times N^{(d-3) / 4}\left(-\frac{D T}{2 \nu}\right)^{N} \cos \left(\sqrt{\frac{N \mathbf{x}^{2}}{\nu T}}+\pi \frac{1-d}{4}\right) .
\end{aligned}
$$

Из (7) видно, что члены ряда при больших $N$ сильно осциллируют на фоне степенного роста $N^{(d-3) / 4}$, при этом ряды теории возмущений являются сходящимися с конечным радиусом сходимости, равным $2 \nu /(D T)$. Число диаграмм обычной теории возмущений, дающих вклад в $G^{[N]}$, возрастает по $N$ факториально. Таким образом, распространенное убеждение, что тип сходимости ряда определяется числом диаграмм (см., например, [3]), неверно.

Интересно, что если мы рассмотрим наш точно решаемый пример в импульсно-частотном представлении, тип сходимости рядов теории возмущений станет принципиально другим. Проще всего убедиться в этом, воспользовавшись MSR-формализмом (4), (5). Разложим выражение для функции отклика в ряд по константе связи:

$$
\begin{aligned}
G^{[N]}\left(x_{2}-x_{1}\right)= & \frac{1}{N !} \int \mathcal{D} \mathbf{V} \mathbf{V}^{N} e^{-\mathbf{V}_{i} D_{i j}^{-1} \mathbf{V}_{j} / 2} \times \\
& \times \int \mathcal{D} \varphi^{\prime} \mathcal{D} \varphi \varphi\left(x_{1}\right) \varphi^{\prime}\left(x_{2}\right)\left(g \varphi^{\prime} \nabla \varphi\right)^{N} e^{-\varphi^{\prime} D_{\xi} \varphi^{\prime} / 2-\varphi^{\prime}\left(\partial_{t}-\nu \Delta\right) \varphi}
\end{aligned}
$$

для краткости мы опустили нормировку. Интеграл по $\mathbf{V}$ сразу вычисляется, с учетом нормировки он равен $(-D)^{N / 2}(N-1)$ !!. Интегралы по $\varphi, \varphi^{\prime}$ выражаются через парную функцию отклика в импульсно-частотном представлении при $g=0$. Таким образом,

$$
G^{[N]}(\mathbf{q}, \omega) \sim(-1)^{N / 2}(N-1) ! ! \frac{\left(\mathbf{q}^{2}\right)^{N / 2}}{\left(i \omega+\nu \mathbf{q}^{2}\right)^{N+1}},
$$

т.е. теперь ряд теории возмущений функции отклика расходится факториально. Легко понять, что зависимость асимптотики от представления объясняется наличием $\nabla$ во взаимодействии.

Итак, тип сходимости ряда определяется не только скоростью роста числа диаграмм теории возмущений, но и существенно зависит от выбранного представления.

Описанный в этом разделе частный случай опровергает подход, при котором тип сходимости ряда определяется числом диаграмм теории возмущений. Тем не менее 
нам неизвестны физические приложения, соответствующие такой редуцированной модели. Поэтому в дальнейшем мы займемся исследованием более реалистичной модели (1), (2). Однако, несмотря на свою простоту, этот частный случай хорошо отражает определенные свойства сходимости рядов физически интересной модели Крейчнана с замороженным полем скорости, в которой, как будет показано ниже, радиус сходимости рядов теории возмущений также оказывается конечным на фоне факториального роста числа диаграмм.

\section{4. ПЕРЕМЕННЫЕ ЛАГРАНЖА}

В работах [7], [14] обсуждалось, что в рамках MSR-формализма инстантон в модели Крейчнана не существует (по крайней мере, в классе гладких убывающих функций). Поэтому, как и в работах [6], [7], введем лагранжевы переменные, т.е. от скалярных полей $\varphi, \varphi^{\prime}$ перейдем к векторным полям $\mathbf{c}(\tau), \mathbf{c}^{\prime}(\tau)$, которые играют роль координат и импульсов жидких частиц и зависят только от времени $(0 \leqslant \tau \leqslant T)$. В этих переменных можно рассматривать различные функции Грина. В настоящей работе мы будем исследовать простейшую из них - функцию отклика, играющую важную роль как в теоретических, так и в экспериментальных исследованиях. Выражения для других функций Грина могут быть исследованы аналогично с помощью приведенного ниже представления.

Итак, функция отклика модели Крейчнана в лагранжевых переменных имеет вид (подробный вывод см. в [7])

$$
G(T, \mathbf{x})=\Theta(T) \frac{\int_{\mathbf{c}(0)=0}^{\mathbf{c}(T)=\mathbf{x}} \mathcal{D} \mathbf{c} \mathcal{D} \mathbf{c}^{\prime} \mathcal{D} \mathbf{V} e^{S^{\mathrm{L}}}}{(4 \pi \nu T)^{d / 2} \int_{\mathbf{c}(0)=0}^{\mathbf{c}(T)=0} \mathcal{D} \mathbf{c} \mathcal{D} \mathbf{c}^{\prime} \mathcal{D} \mathbf{V} e^{\left.S\right|_{g=0} ^{\mathrm{L}}(0)}}
$$

где $T \equiv t-t^{\prime}, \mathbf{x} \equiv \mathbf{x}_{2}-\mathbf{x}_{1}$,

$$
S^{\mathrm{L}}=\int_{0}^{T} d \tau\left(-\nu \mathbf{c}^{\prime 2}+i \mathbf{c}^{\prime}\left[\partial_{\tau} \mathbf{c}-g \mathbf{V}(\mathbf{c})\right]\right)-\frac{1}{2} \int d \mathbf{x} d \mathbf{x}^{\prime} \mathbf{V}_{i}(\mathbf{x}) D_{i j}^{-1}\left(\mathbf{x}-\mathbf{x}^{\prime}\right) \mathbf{V}_{j}\left(\mathbf{x}^{\prime}\right) .
$$

Опущенные аргументы полей здесь и далее предполагаются равными $\tau$, скалярные произведения перемножаемых векторов подразумеваются, граничные условия на поле $\mathbf{c}^{\prime}$ при $\tau=0$ и $\tau=T$ свободные.

Чтобы выделить явно зависимость от переменной $\mathbf{x}$, входящей в граничные условия на поле с, зададим сдвиг поля

$$
\mathbf{c}(\tau)=\overline{\mathbf{c}}(\tau)+\mathbf{x} \frac{\tau}{T}
$$

Тогда интегрирование по $\overline{\mathbf{c}}$ будет производиться по линейному функциональному пространству с нулевыми граничными условиями при $\tau=0, \tau=T$. Тем не менее для упрощения записи, там где это возможно, мы будем выражать полученные результаты через исходное поле $\mathbf{c}(\tau)$. После гауссового интегрирования по полю $\mathbf{V}$ действие преобразуется следующим образом:

$$
\bar{S}^{\mathrm{L}}(g)=\int_{0}^{T} d \tau\left(-\nu \mathbf{c}^{\prime 2}+i \mathbf{c}^{\prime} \partial_{\tau} \mathbf{c}-\frac{g^{2}}{2} \int_{0}^{T} d \tau^{\prime} \mathbf{c}_{i}^{\prime}(\tau) D_{i j}\left(\mathbf{c}(\tau)-\mathbf{c}\left(\tau^{\prime}\right)\right) \mathbf{c}_{j}^{\prime}\left(\tau^{\prime}\right)\right) .
$$

Полученный естественным образом параметр $g^{2} \equiv u$ указывает на то, что разложение ведется по четным степеням $g$. 


\section{5. ИНСТАНТОННЫЙ АНАЛИЗ}

Для выделения $N$-го члена ряда квантово-полевой теории возмущений в работе [2] было предложено пользоваться формулой Коши

$$
G(u)=\sum_{N=0}^{\infty} G^{[N]} u^{N}, \quad G^{[N]}=\frac{1}{2 \pi i} \oint \frac{G(u)}{u^{N+1}} d u .
$$

Интегрирование ведется по замкнутому контуру в комплексной плоскости, охватывающему точку $u=0$.

Для вычисления интегралов по $\mathbf{c}^{\prime}, \mathbf{c}$ и $u$ в $G^{[N]}$ применим метод стационарной фазы. Произведем растяжения

$$
\left\{\overline{\mathbf{c}}, \mathbf{c}^{\prime}\right\} \rightarrow\left\{N^{1 / 2} \overline{\mathbf{c}}, N^{1 / 2} \mathbf{c}^{\prime}\right\}, \quad \mathbf{x} \rightarrow \sqrt{N} \mathbf{x}, \quad u \rightarrow N^{\beta} u .
$$

Учитывая однородность коррелятора скорости по аргументу $\left(D(\mathbf{z}) \sim|\mathbf{z}|^{-2 \beta}\right.$, см. (3)), можно вынести перед действием $\bar{S}^{\mathrm{L}}(g)$ параметр $N$, что упрощает анализ вклада старших вариаций. Такие же растяжения полей $\mathbf{c}, \mathbf{c}^{\prime}$ выполняем и в нормировочном множителе, который для краткости обозначаем $\aleph$. В результате получаем

$$
\begin{gathered}
G^{[N]}(T, \sqrt{N} \mathbf{x})=\frac{N^{-N \beta}}{\aleph} \frac{\Theta(T)}{2 \pi i} \oint \frac{d u}{u} \int_{\overline{\mathbf{c}}(0)=0}^{\overline{\mathbf{c}}(T)=0} \mathcal{D} \mathbf{c} \mathcal{D} \mathbf{c}^{\prime} e^{N S}, \\
S=\int_{0}^{T} d \tau\left(-\nu \mathbf{c}^{\prime 2}+i \mathbf{c}^{\prime} \partial_{\tau} \mathbf{c}-\frac{u}{2} \mathbf{c}_{i}^{\prime}(\tau) \varkappa_{i}(\mathbf{c}(\tau))\right)-\ln u \\
\varkappa_{i}(\mathbf{c}(\tau)) \equiv \int_{0}^{T} d \tau^{\prime} D_{i j}\left(\mathbf{c}(\tau)-\mathbf{c}\left(\tau^{\prime}\right)\right) \mathbf{c}_{j}^{\prime}\left(\tau^{\prime}\right) .
\end{gathered}
$$

Основной вклад в (13) при $N \rightarrow \infty$ дает область интегрирования в окрестности инстантона - значений $\overline{\mathbf{c}}_{\mathrm{st}}, \mathbf{c}_{\mathrm{st}}^{\prime}, u_{\mathrm{st}}$, реализующих экстремум функционала действия, т.е. решений уравнений стационарности. Естественно искать эти решения, предполагая коллинеарность векторов $\mathbf{c}, \mathbf{c}^{\prime}, \mathbf{x}$, так как исходно симметрия задачи нарушена именно вектором $\mathbf{x}$. Данное предположение существенно упрощает структуру действия (14) по векторным аргументам, а коррелятор скорости при этом можно положить равным скалярной функции

$$
D(\mathbf{x})=\frac{D_{0}}{|\mathbf{x}|^{2 \beta}}, \quad D_{0}=a_{1}+a_{2},
$$

где параметры $a_{1}$ и $a_{2}$ определены в приложении Б. Тогда для проекций уравнений стационарности на выделенное направление векторов $\mathbf{c}, \mathbf{c}^{\prime}$ имеем

$$
\begin{array}{cccc}
\frac{\delta S}{\delta c^{\prime}(\tau)}=0 & \Rightarrow & -2 \nu c^{\prime}(\tau)+i \partial_{\tau} c(\tau)=u \varkappa(c(\tau)), \\
\frac{\delta S}{\delta c(\tau)}=0 & \Rightarrow & -i \partial_{\tau} c^{\prime}(\tau)=u c^{\prime}(\tau) \frac{\partial \varkappa(c(\tau))}{\partial c}, \\
\frac{\delta S}{\delta u}=0 & \Rightarrow & u=-\frac{2}{\int_{0}^{T} d \tau c^{\prime}(\tau) \varkappa(c(\tau))} .
\end{array}
$$


Если система (17)-(19) интегродифференциальных уравнений имеет решение инстантон $c_{\mathrm{st}}, c_{\mathrm{st}}^{\prime}, u_{\mathrm{st}}$, причем поля $c_{\mathrm{st}}, c_{\mathrm{st}}^{\prime}$ принадлежат такому функциональному пространству, что функционал действия на них не обращается в бесконечность, то асимптотика корреляционной функции может быть записана в виде

$$
G^{(N)}(T, \sqrt{N} x) \sim N^{-N \beta} e^{N S\left(c_{\mathrm{st}}, c_{\mathrm{st}}^{\prime}, u_{\mathrm{st}}\right)} \Phi(\Delta),
$$

где $\Phi(\Delta)$ - результат функционального интегрирования по флуктуациям в окрестности инстантона

$$
\mathbf{c}=\mathbf{c}_{\mathrm{st}}+\delta \mathbf{c}, \quad \mathbf{c}^{\prime}=\mathbf{c}_{\mathrm{st}}^{\prime}+\delta \mathbf{c}^{\prime}, \quad u=u_{\mathrm{st}}+\delta u
$$

с учетом нормировочного множителя $\aleph$. В результате обычного для инстантонного анализа растяжения флуктуаций $\delta c, \delta c^{\prime}, \delta u$ в $N^{-1 / 2}$ раз расчет главного порядка по $N$ для $\Phi$ сводится к вычислению гауссова интеграла. Кроме того, эти растяжения позволяют явно выделить зависимость этого объекта от $N$. Она сводится к конечной степени $N^{w}$, где параметр $w$ можно определить, вычислив так называемые нулевые моды оператора второй вариации действия на инстантоне.

Отметим, что погрешность выражения, полученного в результате инстантонного подхода, определяется лишь отброшенными старшими вариациями действия на инстантоне (негауссовостью флуктуаций). В случае такого растяжения флуктуаций каждая последующая вариация по сравнению с предыдущей содержит дополнительный множитель $1 / \sqrt{N}$, т.е. соответствующие вклады можно не учитывать при $N \rightarrow \infty$.

Из выражения (20) при $\beta>0$ вытекает сходимость рядов, однако принципиальным остается вопрос: существуют ли вообще решения интегродифференциальной системы (17)-(19) в нужном классе функций? В следующем разделе мы получим такое решение.

\section{6. СУЩЕСТВОВАНИЕ И ЯВНЫЙ ВИД ИНСТАНТОНА}

Покажем, что для системы (17)-(19) удается получить первый интеграл движения.

Воспользовавшись очевидным соотношением

$$
\partial_{\tau} \varkappa(c(\tau))=\frac{\partial \varkappa}{\partial c} \partial_{\tau} c
$$

из уравнений $(17),(18)$ найдем связь $c$ и $c^{\prime}$ : продифференцируем (17) по $\tau$, а равенство (18) умножим на $\partial_{\tau} c$. Исключив $\partial_{\tau} \varkappa(c(\tau))$ из этих двух уравнений, получаем дифференциальное уравнение

$$
-2 \nu \partial_{\tau} c^{\prime}(\tau)+i \partial_{\tau}^{2} c(\tau)+i \frac{\partial_{\tau} c^{\prime}(\tau) \partial_{\tau} c(\tau)}{c^{\prime}(\tau)}=0
$$

Решение этого линейного по $\partial_{\tau} c(\tau)$ уравнения имеет вид

$$
-\nu c^{2}+i c^{\prime} \partial_{\tau} c=i F
$$


где $F$ - интеграл движения. Тем самым вместо системы интегродифференциальных уравнений (17)-(19) можно рассматривать лишь одно интегродифференциальное уравнение, дополненное алгебраическим соотношением (21) и уравнением (19).

Из (19) и (21) очевидно, что первые три слагаемых действия (14) в точке стационарности равны $i F T+1$. K сожалению, вычисление $u_{\text {st }}$ и соответствующего вклада последнего слагаемого (14) при произвольном $F$ возможно лишь численными методами. Хотя принципиальных сложностей при этом не возникает, в настоящей работе мы хотели бы ограничиться лишь аналитическими результатами.

Разным значениям $F$ в $(21)$ соответствуют различные граничные условия на поле $\mathbf{c}$ (различные $\mathbf{x}$ ). Оказывается возможным решить обратную задачу. Зафиксируем интеграл движения $F=0$ и построим частное решение, соответствующее ему. Исследуем, к какому классу функций оно принадлежит и каким граничным условиям соответствует.

Для рассматриваемого частного решения из (21) следует соотношение

$$
\mathbf{c}^{\prime}(\tau)=\frac{i \partial_{\tau} \mathbf{c}(\tau)}{\nu}
$$

Подстановка этого соотношения в (17) дает

$$
-\frac{i}{\nu} \frac{\partial c(\tau)}{\partial \tau}=u \int d \tau^{\prime} D\left(c(\tau)-c\left(\tau^{\prime}\right)\right) \frac{i}{\nu} \frac{\partial c\left(\tau^{\prime}\right)}{\partial \tau^{\prime}}=\frac{i u}{\nu} \int_{0}^{|\mathbf{x}|} d y D(c-y) .
$$

Тогда для поля $c_{\mathrm{st}}$ в квадратурах имеем

$$
\int_{0}^{c} \frac{d y}{\int_{0}^{|\mathbf{x}|} d z D(z-y)}=-\frac{u_{\mathrm{st}} \tau}{\nu}, \quad c \equiv c_{\mathrm{st}}(\tau) .
$$

Величину $u_{\text {st }}$ можно найти, подставив соотношение $(22)$ в (19) с учетом явного вида для $\varkappa$ и $D$. Снова переходя от интегрирования по $\tau \in[0, T]$ к интегрированию по $c \in[0,|\mathbf{x}|]$, получаем

$$
u_{\mathrm{st}}=-\frac{2 \nu^{2}(1-\beta)(2 \beta-1)}{D_{0}|\mathbf{x}|^{2-2 \beta}} .
$$

Для краткости мы не рассматриваем проблемы регуляризации и ренормировки теории, так как принципиальных сложностей на этом этапе не возникает. Полученное нами решение (24) записано в квадратурах для произвольного коррелятора скорости и справедливо при любой регуляризации. В настоящей работе рассматривается степенное поведение коррелятора $D$, что подразумевает аналитическую регуляризацию выражения (24) (напомним, что регуляризация в данной модели как раз и определяется выбором явного вида коррелятора (2)).

Вместо того чтобы определять величину $F$ по переменной х, мы поступили наоборот: положили $F=0$. Посмотрим теперь, какому значению $\mathbf{x}$ соответствует найденное нами частное решение; обозначим это значение $\mathbf{x}_{0}$. Положим в равенстве $(24)$ $\tau=T, \mathbf{c}(T)=\mathbf{x}_{0}$ и, учитывая явный вид коррелятора $D$, вычислим соответствующие интегралы. Получаем

$$
\mathbf{x}_{0}^{2 \beta}=\frac{u_{\mathrm{st}} T D_{0}}{\nu(2 \beta-1) R(\beta)}, \quad R(\beta)=\int_{0}^{1} \frac{d h}{(1-h)^{1-2 \beta}+h^{1-2 \beta}} .
$$


С учетом (25) соотношение (26) можно записать в виде

$$
\mathbf{x}_{0}^{2}=\frac{2(\beta-1)}{R(\beta)} \nu T, \quad u_{\mathrm{st}}=\left(\frac{2(\beta-1)}{R(\beta)} \nu T\right)^{\beta} \frac{\nu(2 \beta-1) R(\beta)}{T D_{0}} .
$$

Итак, на построенном инстантоне действие может быть легко вычислено, и в соответствии с (20) асимптотика величины $G^{[N]}\left(\sqrt{N} \mathbf{x}_{0}\right)$ определена равенством

$$
G^{[N]}\left(T, \sqrt{N} \mathbf{x}_{0}\right) \sim N^{N \beta} N^{w} \frac{e^{N}}{u_{\mathrm{st}}^{N}}
$$

Как уже было отмечено, явное определение численного значения $u_{\mathrm{st}}$ нам удалось провести лишь для некоторого специального значения $\mathbf{x}=\mathbf{x}_{0}$, т.е. лишь на гиперповерхности, на которой выполняется первое из соотношений (27) и где обращается в нуль найденный интеграл движения. Другим значениям х соответствуют другие значения $F$ и, следовательно, другие значения радиуса сходимости $u_{\mathrm{st}}$, для которых мы не можем представить явного выражения и которые могут быть найдены лишь численно. Тем не менее до тех пор пока остается справедливым соотношение (12) (т.е. пока переменная $x$ будет порядка $\sqrt{N}$ ), выражение вида (28) (разумеется, с другими $u_{\text {st }}$ и показателем экспоненты) описывает поведение коэффициентов разложения функции отклика при больших $N$, а именно наличие конечного радиуса сходимости соответствующих рядов.

Например, при малых, но отличных от нуля значениях $F$ можно строить теорию возмущений по $F$, тем самым получая с необходимой точностью инстантон для значений $\mathbf{x}$, лежащих в окрестности $\mathbf{x}_{0}$. Действительно, первая итерация (21) по $F$ дает

$$
c^{\prime}=\frac{i \partial_{\tau} c}{\nu}-\frac{F}{\partial_{\tau} c}
$$

причем в правой части равенства в знаменателе в качестве $\partial_{\tau} c$ достаточно использовать найденное ранее решение при $F=0$. Подстановка первой итерации в (17) дает

$$
-i \partial_{\tau} c-\frac{2 \nu F}{\partial_{\tau} c^{(0)}}=\frac{i u}{\nu} \int_{0}^{x} d y D(c-y)-F u^{(0)} \int_{0}^{x^{(0)}} \frac{D\left(c^{(0)}-y\right) d y}{\left(\partial_{\tau} c^{(0)}\right)^{2}}
$$

здесь индексом (0) отмечены переменные, для которых следует подставлять значение на предыдущем (нулевом) шаге итерации. Это дифференциальное уравнение, как и уравнение (23), с разделяющимися переменными, его решение в квадратурах отличается от выражения (24) наличием в правой части множителя вида $(1+k F)$, где коэффициент $k$ выражается лишь через переменные нулевой итерации. Это позволяет вычислять $\mathbf{x}$ в виде ряда по $F$, а также определять с необходимой точностью величину $u_{\mathrm{st}}$ - радиус сходимости корреляционной функции $(28)$, который будет отличаться от (25) наличием дополнительного множителя - степенного ряда по $F$.

В инстантонном анализе обычной является ситуация, когда асимптотика высоких порядков корреляционной функции существенно неравномерна по аргументу [15]. Аналогичная ситуация наблюдается и в нашем случае. Функция отклика $G(t, \mathbf{x})$ 
имеет существенно неравномерную асимптотику разложений рядов, поскольку из-за проведенного в (12) растяжения переменной х мы определили лишь асимптотику при больших $N$ величины $G^{[N]}(\sqrt{N} \mathbf{x})(28)$. Иными словами, выражение (28) означает, что величина $\left.G^{[N]}\left(t, y_{0}\right)\right|_{y_{0} \equiv \sqrt{N} x_{0}}$ является функцией от $y_{0} / \sqrt{N}, t$ и $N$. Поэтому полученная асимптотика может оказаться неприменимой в области очень больших или очень малых $\mathbf{y}_{0}$, где, вообе говоря, требуются специальные исследования.

Поэтому обсуждать сходимость рядов теории возмущений удобнее для числовых объектов или интегральных характеристик, в которых не проявляется указанная выше неравномерность. Примером такого объекта является функция

$$
\mathcal{G}(T, \gamma)=\int d \mathbf{x} G(T, \mathbf{x}) e^{-\gamma \mathbf{x}^{2}}
$$

Это производящий функционал четных моментов функции отклика. Наличие в $\mathcal{G}$ дополнительного интеграла по $\mathbf{x}$ и упомянутое растяжение переменной $\mathbf{x}$ в $\sqrt{N}$ раз позволяют включить величину $\mathbf{x}$ в рассмотрение в рамках метода стационарной фазы. При этом уже не требуется растягивать переменную $\gamma$ для выделения общего $N$ в действии, это достигается растяжением х. Однако в дополнение к системе (17)-(19) следует рассматривать уравнение стационарности

$$
\frac{\delta\left(S-\gamma \mathbf{x}^{2}\right)}{\delta|\mathbf{x}|}=0 \quad \Rightarrow \quad \int_{0}^{T} d \tau\left(\frac{i c^{\prime}(\tau)}{T}-\frac{u c^{\prime}(\tau)}{2} \frac{\partial \varkappa(\mathbf{c}(\tau))}{\partial|\mathbf{x}|}\right)=2 \gamma|\mathbf{x}|
$$

Подстановка в уравнение (29) частного решения (22), учет явного вида $\varkappa(15)$ и четности коррелятора $D$ приводят (29) к виду

$$
-\frac{u D_{0}}{\nu^{2}(2 \beta-1)|\mathbf{x}|^{2 \beta-1}}=2 \gamma|\mathbf{x}|
$$

Из равенств (26) и (30) очевидно, что, выбирая

$$
\gamma=\gamma_{0} \equiv-\frac{R(\beta)}{2 \nu T}
$$

можно удовлетворить обоим этим уравнениям. Тем самым мы показали, что полученное нами частное решение при $F=0$ является инстантоном, позволяющим определить тип сходимости рядов теории возмущений для функции $\mathcal{G}(T, \gamma)$ в точке $\gamma=\gamma_{0}$

Подставляя $\mathbf{x}_{0}$ из $(26)$ в $(25)$, окончательно получаем

$$
\mathcal{G}^{[N]}\left(T, \gamma_{0}(T)\right) \sim \frac{\Theta(T)}{\aleph(T)}\left[-\frac{2 \nu^{2} \gamma_{0}(2 \beta-1)}{D_{0}}\right]^{N+1}\left[\frac{1-\beta}{\gamma_{0}}\right]^{(N+1) \beta} .
$$

В формуле (31) мы не учитывали множитель $N^{w}$, определив лишь величину радиуса сходимости рассматриваемого ряда. Параметр $w$ определяет тип особенности ряда на границе круга сходимости и может быть найден из гауссового интегрирования по флуктуациям в окрестности инстантона. 


\section{7. ВЫВОДЫ}

Мы рассмотрели асимптотики высоких порядков модели Крейчнана с замороженным полем скорости, построили семейство инстантонов в этой модели и нашли один из них в явном виде. Мы убедились, что соответствующие ряды теории возмущений имеют конечный радиус сходимости, который в отдельных случаях становится бесконечным.

Свойства сходимости рядов данной модели могут быть использованы при их пересуммировании для улучшения численных значений, получаемых с помощью теории возмущений.

Кроме того, мы опровергли широко распространенное убеждение, что ряды оказываются асимптотическими, если число диаграмм в высоких порядках теории возмущений растет факториально, и показали, что тип сходимости зависит от выбранного представления и поведения коррелятора.

Точно решаемый частный случай иллюстрирует наши расчеты и подтверждает наши результаты.

Как уже упоминалось, в настоящей работе мы не обсуждали регуляризацию и ренормировку теории. Асимптотики высоких порядков констант ренормировки рассматриваемой модели предполагается обсудить в следующей статье.

ПРИЛОЖЕНИЕ А

\section{Иллюстрация метода в точно решаемом случае}

Точно решаемый случай $D=D_{0}=$ const соответствует $\beta=0$ (см. (3)). При этом результат (31) дает для производящего функционала $\mathcal{G}$ такой же радиус сходимости ряда $2 \nu /(D T)$, как и точное решение (7) для функции отклика.

Однако в разделе 6 мы ограничились явным вычислением только одного решения из семейства инстантонов, соответствующего лишь фиксированному значению $\mathbf{x}_{0}^{2}$ и построили на основании этого свою схему. В точно решаемом случае мы вычислим все семейство явно и убедимся, что проведенные растяжения полей в (12) полностью отражают поведение всего семейства по параметру $N$. Также мы учтем вклад гауссова интегрирования по флуктуациям в окрестности инстантона, при этом найдем не только радиус сходимости, но и тип особенности на границе круга сходимости. Ответ, который мы получим в этом приложении, будет совпадать как с точным ответом (7), так и с формулой для радиуса сходимости, полученной в разделе 6 для случая $\beta=0$. Тем самым мы еще раз убедимся, что инстантонный анализ для рядов функции $\mathcal{G}$ не отличается принципиально от анализа рядов функции отклика.

Найдем зависимость переменных от $N$ явно, не предполагая растяжений (12). Проекция вариационного уравнения по полю с на выделенное направление при этом имеет тот же вид, что и $(18)$, и при $D=D_{0}$ и $\varkappa(\mathbf{c})=$ const. Это уравнение сразу приводит к независящему от времени полю $\mathbf{c}^{\prime}$. Вариации по $\mathbf{c}^{\prime}$ снова дают уравнение (17), из которого теперь следует, что поле с также не зависит от времени и

$$
\overline{\mathbf{c}} \equiv 0, \quad \mathbf{c}^{\prime}=\frac{i \mathbf{x}}{2 \nu T+u D_{0} T^{2}} .
$$

Вариационное уравнение по $u$ (ср. с (19)) теперь имеет вид

$$
\frac{\delta S}{\delta u}=0 \quad \Rightarrow \quad u=-\frac{2 N}{D_{0} c^{\prime 2} T^{2}} .
$$


Совместное решение (32) и (33) дает

$$
\begin{aligned}
& c_{\mathrm{st}}^{\prime}= \pm \sqrt{\frac{N}{\nu T}}+\frac{i|\mathbf{x}|}{4 \nu T}+O\left(N^{-1 / 2}\right), \\
& u_{\mathrm{st}}=-\frac{2 \nu}{D T} \pm \frac{i|\mathbf{x}| \sqrt{\nu T}}{D T^{2}} \frac{1}{\sqrt{N}}+\frac{|\mathbf{x}|^{2}}{4 D T^{2}} \frac{1}{N}+O\left(N^{-3 / 2}\right),
\end{aligned}
$$

т.е. две точки стационарности. Подставляя полученные значения в действие (14) (без растяжений переменных по $N$ ), получаем, что в точке стационарности в главном порядке по $N$ оно имеет вид

$$
S_{0}^{( \pm)}=-N \ln \left[-\frac{2 \nu}{D T}\right] \pm i \mathbf{x} \sqrt{\frac{N}{\nu T}}
$$

Инстантонный анализ для функции отклика дает

$$
G^{[N]} \sim \Theta(T) \int d \delta u \int \mathcal{D} \delta \mathbf{c} \mathcal{D} \delta \mathbf{c}^{\prime}\left(e^{S_{0}^{(+)}+S_{2}^{(+)}}+e^{S_{0}^{(-)}+S_{2}^{(-)}}\right),
$$

где $\delta \mathbf{c}, \delta \mathbf{c}^{\prime}, \delta g$ - флуктуации полей в окрестности инстантона, $S_{2}^{( \pm)}$- вторая вариация действия $S$ в соответствующих точках стационарности:

$$
\begin{aligned}
S_{2}= & \int_{0}^{T} d \tau\left(-\nu \delta \mathbf{c}^{\prime 2}(\tau)+i \delta \mathbf{c}_{i}^{\prime}(\tau) \partial_{\tau} \delta \mathbf{c}_{i}(\tau)-\left.\delta u \delta \mathbf{c}_{i}^{\prime}(\tau) c_{i}^{\prime}\right|_{\mathbf{c}^{\prime}=\mathbf{c}_{\mathrm{st}}^{\prime}} D_{0} T\right)+ \\
& +\frac{N}{u_{\mathrm{st}}^{2}} \delta u^{2}-u_{\mathrm{st}} D_{0} \frac{1}{2} \int_{0}^{T} \int_{0}^{T} d \tau d \tau^{\prime} \delta \mathbf{c}_{i}^{\prime}(\tau) \delta \mathbf{c}_{i}^{\prime}\left(\tau^{\prime}\right)
\end{aligned}
$$

Заметим, что во все слагаемые, кроме третьего, дают вклад как продольные, так и поперечные по отношению к вектору х вариации полей. В третьем же слагаемом имеется лишь продольный вклад, поскольку поле $\delta \mathbf{c}^{\prime}$ свернуто с полем $\mathbf{c}_{\mathrm{st}}^{\prime} \| \mathbf{x}$.

Таким образом, после растяжения $\delta u$ в $\sqrt{N}$ раз и вычисления гауссова интеграла по $\delta u$ (при этом возникает множитель $1 / \sqrt{N}$ ) вклады продольных и поперечных компонент поля оказываются разными. Поэтому при вычислении гауссова интеграла от $d-1$ поперечных компонент с учетом нормировки получаем вклад

$$
\begin{aligned}
\int \mathcal{D} & \delta \mathbf{c} \\
\sim & \mathcal{D} \delta \mathbf{c}^{\prime} \exp \left(-\nu \delta \mathbf{c}^{\prime 2}+i \delta \mathbf{c}^{\prime} \partial_{\tau} \delta \mathbf{c}-\frac{u_{\mathrm{st}} D_{0} \delta \mathbf{c}^{\prime} \delta \mathbf{c}^{\prime}}{2}\right) \sim \\
& {\left[\frac{1}{4 \pi T\left(\nu+u_{\mathrm{st}} D_{0} T / 2\right)}\right]^{(d-1) / 2} \sim\left(e^{\mp i \pi} N\right)^{(d-1) / 4} }
\end{aligned}
$$

Зависимость от $N$ появляется из-за того, что в силу (35) величина $\left(\nu+u_{\mathrm{st}} D_{0} T / 2\right)$ мала. Аналогичный же вклад для продольных вариаций оказывается порядка $O(1)$.

Окончательно, инстантонный анализ для $G^{[N]}$ дает

$$
G^{[N]} \sim \Theta(T)\left(-\frac{D T}{2 \nu}\right)^{N} N^{(d-3) / 4} \cos \left(\sqrt{\frac{N \mathbf{x}^{2}}{\nu T}}+\pi \frac{1-d}{4}\right),
$$

что в старшем порядке по $N$ ведет себя при больших $N$ так же, как (7). 
ПРИЛОЖЕНИЕ Б

Параметры коррелятора скорости имеют следующие значения:

$$
\begin{aligned}
a_{1} & =\frac{\Gamma(\beta)}{2^{2 \alpha+1} \pi^{d / 2} \Gamma(\alpha+1)}\left(\lambda_{\mathrm{T}}(2 \alpha-1)+\lambda_{\mathrm{L}}\right), \\
a_{2} & =\left(\lambda_{\mathrm{T}}-\lambda_{\mathrm{L}}\right) \frac{\Gamma(\beta+1)}{2^{2 \alpha} \pi^{d / 2} \Gamma(\alpha+1)},
\end{aligned}
$$

где $\lambda_{\mathrm{T}}$ и $\lambda_{\mathrm{L}}-$ продольная и поперечная константы связи.

$G^{[N]}$ в точно решаемом случае имеет вид

$$
G^{[N]}=\frac{(-1)^{N} \Theta(T) \Gamma(d / 2+N)}{\Gamma(d / 2) N !}{ }_{1} F_{1}\left(\frac{d}{2}+N, \frac{d}{2},-\frac{\mathbf{x}^{2}}{4 \nu T}\right) .
$$

Благодарности. Работа выполнена при финансовой поддержке РФФИ (грант № 05-02-17524).

\section{Список литературы}

[1] J. Zinn-Justin, Quantum Field Theory and Critical Phenomena, Internat. Ser. Monogr. Phys., 77, Oxford Univ. Press, Oxford, 1989.

[2] Л.Н. Липатов, ЖЭТФ, 72:2 (1977), 411-427.

[3] S. A. Orszag, V. Yakhot, J. Sci. Comput., 14:2 (1999), 147-195.

[4] J. Honkonen, M. V. Komarova, M. Yu. Nalimov, Nucl. Phys. B, 707:3 (2005), 493-508.

[5] J. Honkonen, M. V. Komarova, M. Yu. Nalimov, Nucl. Phys. B, 714:3 (2005), 292-306.

[6] M. Chertkov, Phys. Rev. E, 55:3 (1997), 2722-2735.

[7] A. Yu. Andreanov, M. V. Komarova, M. Yu. Nalimov, J. Phys. A, 39:25 (2006), 7801-7813.

[8] J. Konkonen, E. Karjalainen, J. Phys. A, 21:22 (1988), 4217-4234.

[9] J. Honkonen, E. Karjalainen, Phys. Lett. A, 129:5-6 (1988), 333-338.

[10] А.Н. Васильев, Квантовополевая ренормгруппа в теории критического поведения и стохастической динамике, ПИЯФ, СПб., 1998.

[11] J. P. Bouchaud, A. Georges, Phys. Rep., 195:4-5 (1990), 127-293.

[12] P. C. Martin, E. D. Siggia, H. A. Rose, Phys. Rev. A, 8:1 (1973), 423-437.

[13] И.С. Градштейн, И.М. Рыжик, Таблицы интегралов, сумм, рядов и произведений, Физматлит, М., 1963.

[14] E. Balkovsky, V. Lebedev, Phys. Rev. B, 58:5 (1998), 5776-5795.

[15] М.В. Комарова, М. Ю. Налимов, ТМФ, 129:3 (2001), 387-402.

Поступила в редакцию 17.01.2008, после доработки 6.06.2008 